\title{
Extended finite element method for three-dimensional crack modelling
}

\author{
N. Sukumar ${ }^{\ddagger}$, N. Moës ${ }^{\S}$, B. Moran ${ }^{\llbracket}$ and T. Belytschko*,+,\| \\ Department of Civil and Mechanical Engineering, Northwestern University, 2145 Sheridan Road, \\ Evanston, IL 60208, U.S.A.
}

\begin{abstract}
An extended finite element method (X-FEM) for three-dimensional crack modelling is described. A discontinuous function and the two-dimensional asymptotic crack-tip displacement fields are added to the finite element approximation to account for the crack using the notion of partition of unity. This enables the domain to be modelled by finite elements with no explicit meshing of the crack surfaces. Computational geometry issues associated with the representation of the crack and the enrichment of the finite element approximation are discussed. Stress intensity factors (SIFs) for planar three-dimensional cracks are presented, which are found to be in good agreement with benchmark solutions.
\end{abstract}

KEY WORDS: extended finite element method; partition of unity; local enrichment; elastostatics; planar threedimensional cracks

\section{INTRODUCTION}

The accurate modelling of three-dimensional cracks in finite bodies remains a challenging problem in computational mechanics. The relevance and importance of the computation of fracture parameters and the simulation of three-dimensional crack growth stems from the widespread use of numerical fracture mechanics in fatigue life predictions of safety-critical components such as aircraft fuselages, pressure vessels, automobile components, and castings. Fatigue failure usually

\footnotetext{
${ }^{*}$ Correspondence to: T. Belytschko, Department of Mechanical Engineering, Northwestern University, 2145 Sheridan Road, Evanston, IL 60208, U.S.A.

†E-Mail: t-belytschko@nwu.edu

¥Post-Doctoral Research Fellow, Theoretical and Applied Mechanics

$\S$ Research Associate, Department of Mechanical Engineering

ฯAssociate Professor of Civil Engineering

" Walter P. Murphy, Professor of Computational Mechanics

Contract/grant sponsor: National Science Foundation; contract/grant number: CMS-9732319

Contract/grant sponsor: Georgia Institute of Technology ONR; contract/grant number: N0014-95-1-0539

Contract/grant sponsor: Federal Aviation Administration; contract/grant number: DTFA03-98-F-IA025

Contract/grant sponsor: Office of Naval Research
} 
occurs due to the initiation and propagation of surface or near-surface cracks, which are often assumed to be elliptical or semi-elliptical in shape for numerical modelling. Closed-form solutions for the stress intensity factors (SIFs) are available for simple crack geometries in three dimensions; however, for arbitrary-shaped cracks in finite specimens, numerical methods are the only recourse to modelling three-dimensional fatigue crack growth.

Currently, finite element methods based on non-singular as well as singular elements are widely used in linear elastic fracture analysis. Methods based on singular elements [1-3] are able to provide accurate stress intensity factors. Enriched element methods which incorporate the asymptotic crack-tip fields in the trial functions $[4,5]$ provide stress intensity factors directly as part of the solution. In spite of the successes using finite elements in computational fracture, mesh generation in three dimensions is time consuming and especially burdensome for multiple crack configurations and crack growth simulations. It is difficult to explicitly model the crack topology as part of the finite element since accuracy considerations require significant refinement in the vicinity of the crack front.

The modelling of growing cracks using finite elements and boundary elements with re-meshing has been pursued by many researchers [6-8]. The finite element alternating method which is based on the Schwartz-Neumann alternating method has been applied to the modelling of arbitrary crack configurations in three dimensions $[9,10]$. Numerical techniques such as the boundary integral equation method [11], body force method [12], and the self-similar crack expansion method [13] also enable the accurate computation of stress intensity factors for three-dimensional cracks. The requirement of a Green's function imposes restriction on the scope of application of the boundary integral equation based methods. These methods are not readily extendable to non-linear problems nor to the study of cracks in anisotropic materials. The modelling of cracks as a continuous distribution of infinitesimal dislocation loops has also gained prominence. In the dislocation distribution approach, the crack surface in three dimensions is assumed to be made up of a continuous distribution of infinitesimal dislocation loops with the Burger's vector corresponding to the jump in displacement across the crack surface; a hypersingular integral equation is obtained on the crack surface which is solved in the Hadamard finite-part sense $[14,15]$. More recently, the element-free Galerkin (EFG) method, a mesh-free method, has been successful in modelling static and dynamic fracture in two dimensions [16, 17] and three dimensions [18, 19].

The extended finite element method (X-FEM) alleviates shortcomings associated with meshing of the crack surfaces in existing methods. The finite element method is used as the building block in the extended finite element method, and hence much of the theoretical and numerical developments in finite elements can be readily extended and applied. In this paper, the finite element approximation is enriched by additional functions through the notion of partition of unity [20]. Strouboulis et al. [21] used local enrichment functions within the partition of unity framework for modelling re-entrant corners in two dimensions, and in Reference [22], enrichment functions for holes are proposed. In X-FEM, the additional functions are used to model the presence of cracks, voids or inhomogeneities, and also to improve accuracy in problems where some aspects of the functional behaviour of the solution field is known a priori. For crack modelling, a discontinuous function and the two-dimensional asymptotic crack-tip displacement fields are added to the displacement-based finite element approximation. Partition of unity enrichment methods for discontinuities and near-tip crack fields were introduced by Belytschko and Black [23]. Moës et al. [24] proposed the generalized Heaviside function as a means to model the crack away from the crack tip, and developed simple rules for the introduction of the discontinuous and crack-tip enrichments. The numerical results presented in this paper demonstrate that for three-dimensional 
cracked bodies, the accuracy and performance of X-FEM is excellent. This opens up many exciting possibilities for its further development and advancement.

The outline of this paper follows. In the following section, we introduce the extended finite element method, and in Section 3, we describe crack modelling in $\mathbb{R}^{2}$ and $\mathbb{R}^{3}$. In Section 4, the computational methodology adopted in X-FEM and the numerical issues involved are discussed. The strong and weak forms of the Galerkin method for elastostatics, along with the discrete equations for X-FEM are given in Section 5. In Section 6, a brief description of three-dimensional domain integral computations is presented, which is followed by numerical results for several problems in three-dimensional linear elastic fracture mechanics. The numerical SIF results are compared to available reference solutions from the literature. Some final remarks and conclusions are made in Section 7.

\section{EXTENDED FINITE ELEMENT METHOD}

In finite element methods, a partition of a domain into sub-domains (elements) forms the basis of mesh generation. The presence of flaws or inhomogeneities such as cracks, voids, and inclusions must be taken into account in mesh generation - the mesh must conform to these geometric entities. In unstructured mesh generation, a Delaunay tessellation of the domain results in triangular elements in $\mathbb{R}^{2}$ and tetrahedral elements in $\mathbb{R}^{3}$. Alternatively, algorithms for the construction of quadrilateral (in two dimensions) or hexahedral meshes (in three dimensions) are also available. In computational fracture, quadrilateral or hexahedral elements are preferred to triangular or tetrahedral elements due to the higher-order approximation in the former. However, in comparison to unstructured mesh generation, hexahedral mesh generation has not reached a mature stage of development, and hence mesh generation in three dimensions for computational fracture applications continues to present many challenges.

The extended finite element method alleviates much of the burden associated with mesh generation by not requiring the finite element mesh to conform to cracks, and in addition, provides a seamless means to use higher-order elements or special finite elements without significant changes in the formulation. The essence of X-FEM lies in sub-dividing a model problem into two distinct parts: mesh generation for the geometric domain (cracks not included), and enriching the finite element approximation by additional functions that model the flaw(s) and other geometric entities.

The enrichment of the finite element approximation is described as follows. Consider a point $\mathbf{x}$ of $\mathbb{R}^{d}(d=1-3)$ that lies inside a finite element $e$. Denote the nodal set $\mathbf{N}=\left\{n_{1}, n_{2}, \ldots, n_{m}\right\}$, where $m$ is the number of nodes of element $e .(m=2$ for a linear one dimensional finite element, $m=3$ for a constant-strain triangle, $m=8$ for a trilinear hexahedral element, etc.) The enriched displacement approximation for a vector-valued function $\mathbf{u}(\mathbf{x}): \mathbb{R}^{d} \rightarrow \mathbb{R}^{d}$ assumes the form

$$
\mathbf{u}^{h}(\mathbf{x})=\sum_{\substack{I \\ n_{I} \in \mathbf{N}}} \phi_{I}(\mathbf{x}) \mathbf{u}_{I}+\sum_{\substack{J \\ n_{J} \in \mathbf{N}^{g}}} \phi_{J}(\mathbf{x}) \psi(\mathbf{x}) \mathbf{a}_{J}, \quad\left(\mathbf{u}_{I}, \mathbf{a}_{J} \in \mathbb{R}^{d}\right)
$$

where the nodal set $\mathbf{N}^{g}$ is defined as

$$
\mathbf{N}^{g}=\left\{n_{J}: n_{J} \in \mathbf{N}, \omega_{J} \cap \Omega_{\mathrm{g}} \neq \emptyset\right\}
$$

In the above equation, $\omega_{J}=\operatorname{supp}\left(n_{J}\right)$ is the support of the nodal shape function $\phi_{J}(\mathbf{x})$, which consists of the union of all elements with $n_{J}$ as one of its vertices; and $\Omega_{\mathrm{g}}$ is the domain associated 


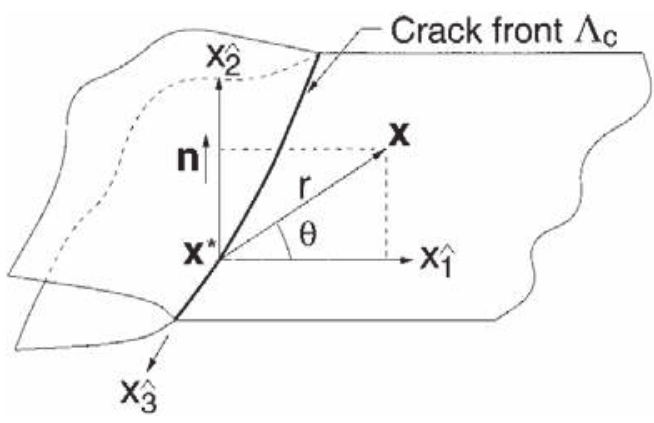

Figure 1. Co-ordinate configuration for crack front enrichment functions.

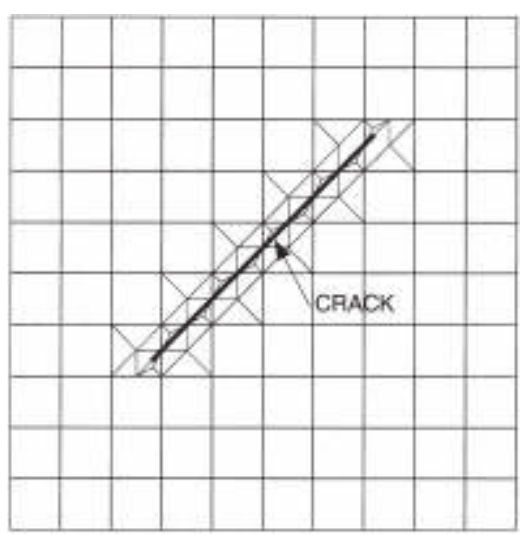

Figure 2. Partitioning algorithm for a crack in two dimensions.

with a geometric entity such as a hole, crack surface, or crack front. In general, the choice of the function $\psi(\mathbf{x})$ depends on the geometric entity.

\section{CRACK MODELLING}

The crack is modelled by enriching the nodes whose nodal shape function support intersects the interior of the crack by a discontinuous function, and enriching the nodes whose nodal shape function support intersects the crack front (crack-tip in $\mathbb{R}^{2}$ ) by the two-dimensional asymptotic crack-tip fields.

\subsection{Enrichment}

We restrict the decription of the implementation to planar cracks in three dimensions. The enrichments concepts that follow are easily extended to non-planar cracks but the implementation is more difficult.

Consider a single crack in three dimensions, and let $\Gamma_{\mathrm{c}}$ be the crack surface and $\Lambda_{\mathrm{c}}$ the crack front. Note that for an internal crack, the crack front corresponds to the boundary of the crack: $\Lambda_{\mathrm{c}}=\partial \Gamma_{\mathrm{c}}$ whereas for an edge crack, the crack front is only part of the boundary: $\Lambda_{\mathrm{c}} \subset \partial \Gamma_{\mathrm{c}}$. The interior of a planar crack is modelled by the enrichment function $H(\mathbf{x})$, which we refer to as a generalized Heaviside function. The function $H(\mathbf{x})$ takes on the value +1 above the crack and -1 below the crack. More precisely, let $\mathbf{x}^{*}$ be the closest point to $\mathbf{x}$ on the crack $\Gamma_{\mathrm{c}}$, and $\mathbf{n}$ be the normal to the crack plane (Figure 1$)$. The $H(\mathbf{x})$ function is then given by +1 if $\left(\mathbf{x}-\mathbf{x}^{*}\right) \cdot \mathbf{n} \geqslant 0$ and -1 otherwise, i.e.

$$
H(\mathbf{x})= \begin{cases}1 & \text { if }\left(\mathbf{x}-\mathbf{x}^{*}\right) \cdot \mathbf{n} \geqslant 0 \\ -1 & \text { otherwise }\end{cases}
$$

To model the crack front and also to improve the representation of crack-tip fields in threedimensional computations, crack-tip enrichment functions are used in elements which contain the crack front. In the neighbourhood of the crack front, the asymptotic fields are two-dimensional in 
nature. The crack front enrichment consists of functions which incorporate the radial and angular behaviour of the two-dimensional asymptotic crack-tip displacement field:

$$
\boldsymbol{\Psi}(\mathbf{x}) \equiv\left\{\psi_{1}, \psi_{2}, \psi_{3}, \psi_{4}\right\}=\left[\sqrt{r} \cos \frac{\theta}{2}, \sqrt{r} \sin \frac{\theta}{2}, \sqrt{r} \sin \theta \sin \frac{\theta}{2}, \sqrt{r} \sin \theta \cos \frac{\theta}{2}\right]
$$

where $r$ and $\theta$ are polar co-ordinates in the $x_{\hat{1}}-x_{\hat{2}}$ plane (Figure. 1). Note that the second function in the above equation is discontinuous on the crack plane.

If a three-dimensional planar crack coincides with element boundaries, an equivalence exists between the finite element space with the crack explicitly modelled by the mesh and the X-FEM space with $H(\mathbf{x})$ used as the enrichment function. This correspondence for two-dimensional cracks was shown in References [24, 25], which readily extends to three dimensions.

\section{COMPUTATIONAL METHODOLOGY}

Computational geometry issues associated with the representation of the crack and the enrichment of the finite element approximation are discussed. The X-FEM implementation is performed in $\mathrm{C}^{++}$: the object-oriented features of $\mathrm{C}^{++}$provide for better data management, encapsulation, code re-usability, flexibility, and maintenance. In the following sub-section, we describe some of the important computational geometry issues, with an aim towards an accurate and robust implementation.

\subsection{Mesh-geometry interactions}

The finite element mesh consists of tetrahedral, prismatic, and hexahedral elements. In two dimensions, cracks are represented by line segments and the crack front is a point. In three dimensions, a crack is represented by a polygon partition into triangles, and the crack front consists of line segments as in Reference [19].

The notion of geometric predicates which is widely used in computational geometry, is also an integral part of the X-FEM mesh-geometric computations. In the following sub-section, we briefly touch upon the concept of geometric predicates. In crack modelling, different functions are used to enrich the displacement approximation in the interior of the crack and on the crack front. Therefore, one of the first tasks is to determine the finite elements that intersect the crack. These finite elements are then partitioned into simplices so that numerical integration of the weak form accounts for the discontinuities on either side of the crack surface. These sub-tetrahedrons are also used to determine whether a node is to be enriched (see Section 4.1.4).

4.1.1. Geometric predicates. Since data is stored and computations performed using finite-precision arithmetic, it is essential that the robustness of algorithms is maintained even for small perturbations in the data. Efforts have been made to develop robust geometric predicates [26], which are especially important in the development of algorithms for the Delaunay tessellation and Voronoi diagram of a point set.

The incircle and orientation tests are widely used in computational geometry. The orientation test determines whether a point lies to the left of, to the right of, or on a line or plane defined by other points. The incircle test determines whether a point lies inside, outside, or on a circle (sphere) defined by other points. Each of these tests is performed by evaluating the sign of a 
determinant. Instead of explicitly computing intersections, geometric predicates provide an easier and robust means to evaluate queries.

In X-FEM, the orientation test is used to determine the nodal enrichment for a query point $\mathbf{x}$ and in the algorithm for the partitioning of the finite elements into tetrahedrons in three dimensions. In the evaluation of the predicates associated with a planar crack and for generality to both two and three dimensions, we assume the crack plane (crack segment in two dimensions) is defined by a point $\mathbf{x}_{0}$ that lies on it and a unit normal $\mathbf{m}$. In two dimensions, if $\mathbf{e}_{1}$ and $\mathbf{e}_{2}$ are unit vectors in the plane, then $\mathbf{t} \times \mathbf{m}$ is a vector along $\mathbf{e}_{3}$, where $\mathbf{t}$ is the tangent vector to the crack segment, and in three dimensions $\mathbf{m}$ is the unit normal to the plane of the crack. The equation of the crack segment in $\mathbb{R}^{2}$ or the crack plane in $\mathbb{R}^{3}$ can be written as

$$
f(\mathbf{x})=\left(\mathbf{x}-\mathbf{x}_{\mathbf{0}}\right) \cdot \mathbf{m}=0
$$

For a query point $\mathbf{x}$, the ternary predicates $\operatorname{ABOVE}(f(\mathbf{x})>\varepsilon)$, BELOW $(f(\mathbf{x})<-\varepsilon)$, and ON $(-\varepsilon \leqslant f(\mathbf{x}) \leqslant \varepsilon)$ are used, where $\varepsilon=10^{-6}$ is used as the tolerance for a finite element mesh with element edge length of $\mathcal{O}(1)$.

4.1.2. Crack-mesh intersection. A naïve approach to finding all finite elements that intersect the crack is to test for intersection with each element in the mesh. Clearly, this is of $\mathcal{O}(N)$ complexity, where $N$ is the number of elements in the mesh, and hence is not a computationally attractive choice. A more appealing alternative that is computationally feasible is adopted. Since determining the elements that intersect with the crack is non-trivial, we first find a candidate set that is possibly larger than the desired set. We use a bounding box $(\mathrm{BB})$ of cell dimensions $10 \times 10 \times 10$ that encompasses the entire finite element mesh. The cell dimensions of the BB-mesh is used as a compromise between computational costs and storage requirements. For a given query point $\mathbf{x}$ that is contained in a BB-element $b$, all the associated finite elements $t_{b}$ that intersect $b$ are obtained. In three dimensions we use a map of the vertices of a triangle from $\mathbf{x} \rightarrow[\xi, \eta]$, where $(0,0),(1,0)$, and $(0,1)$ are the vertex co-ordinates of the reference right-angled triangle. The starting search path for a triangle $\left[\mathbf{x}_{a}, \mathbf{x}_{b}, \mathbf{x}_{c}\right]$ is $(\xi, \eta)=(0,0),(\xi, \eta)=(1,0)$, and $(\xi, \eta)=(0,1)$, In order to find the set of all intersected elements (may be larger than the exact set), a recursive algorithm is implemented. The stopping criterion is

1. if all points are in the same finite element; or

2. if the element sets for all adjacent points have at least one element in common; or

3 . if the distance between all points in the reference co-ordinate is less than a prescribed tolerance. In three dimensions, $\left|\xi_{1}-\xi_{2}\right|<10^{-5} / \sqrt{V}$ and $\left|\eta_{1}-\eta_{2}\right|<10^{-5} / \sqrt{V}$, where $V$ is the area of the triangle (simplex partition of the crack).

4.1.3. Partitioning of finite elements. The algorithm to compute the intersected finite elements is outlined in Section 4.1.2. Here we describe the algorithm to partition a given three-dimensional finite element into tetrahedrons. The partitioning is carried out for all the elements that are selected on the basis of the algorithm presented in Section 4.1.2; as mentioned earlier, this set of elements can be larger than the exact set of elements that intersect the crack domain. In Section 4.1.4, an additional criterion is invoked so that the nodal enrichment is precise.

We first illustrate the procedure in two dimensions. Each crack segment is defined by a point $\mathbf{x}_{0}$ on it and the unit normal vector $\mathbf{m}$ that is defined in the previous sub-section. The face and element connectivity of each finite element are also known, with counterclockwise orientation of 
the nodes assumed in the connectivity. Standard Template Library (STL) containers in $\mathrm{C}^{++}$are used to create maps for node-to-co-ordinates, node-to-orientation, and edge-to-connectivity for the finite element. The orientation $(\mathrm{ABOVE}=+1, \mathrm{BELOW}=-1, \mathrm{ON}=0)$ of the nodes of the finite element are set and stored in the node-to-orientation map.

The element is partitioned if and only if there exists two nodes in the connectivity with orientation +1 and -1 . A loop over the edges of the finite element is carried out, and if there is an intersection for an edge, the point of intersection is computed and added to the node-to-co-ordinate and edge-to-connectivity maps. The orientation of the point of intersection is set to +10 if the orientation of the first point of the edge is +1 , and -10 if the orientation of the first point is -1 . This updates the edge map for the finite element. Now, using the edge map of the finite element, two new edge maps for the domains above and below the crack are created. The two edges (upper and lower) that correspond to the crack segment are also created such that counterclockwise orientation of the nodes is preserved. The centroid of the two domains is evaluated with the orientation for the node that belongs to the surface above the crack set to +100 , and the orientation set to -100 for the node that belongs to the surface below the crack. A loop over the two edge maps is carried out and on using the centroid for each surface, the partitioned elements are obtained. The partitioned elements for a sample crack are illustrated in Figure 2.

The partitioning of three-dimensional polyhedrons is more involved than the two-dimensional case. Even for the simplified case of partitioning a three-dimensional finite element that is cut by a plane, no public-domain package that can readily interface to a $\mathrm{C}^{++}$program is currently available. An algorithm was hence developed and implemented for the partitioning of a threedimensional finite element (such as tetrahedron, prism, or hexahedron) by a single crack plane. The crack is defined by a point $\mathbf{x}_{0}$ that lies on it and the unit normal vector $\mathbf{m}$ that is defined in the previous sub-section. The nodal connectivities of the finite element are stored in counterclockwise orientation, and the face connectivities are stored such that an edge that is common to two faces is traversed in opposite directions. The nodal and face connectivity of a tetrahedron are such that if the nodes on a face are traversed in a certain sense, then the thumb, using the right-hand rule, points in the direction of the fourth node. Standard Template Library (STL) containers are used to create maps for node-to-co-ordinates, node-to-orientation, and face-to-connectivity for the finite element. The orientation $(\mathrm{ABOVE}=+1, \mathrm{BELOW}=-1$, ON $=0)$ of the nodes of the finite element are set and stored in the node-to-orientation map.

The element is partitioned if there exists two nodes in the connectivity with orientation +1 and -1 . A loop over the faces of the finite element is carried out, and if the crack plane intersects an edge, the point of intersection is computed and added to the node-to-co-ordinate and face-toconnectivity maps. The orientation of the point of intersection is set to +10 if the orientation of the first point on the edge is +1 , and -10 if the orientation of the first point is -1 . This updates the face-to-connectivity map for the finite element.

Next, using the face map of the finite element, two new maps for the domains above and below the crack plane are created. The two faces (upper and lower) that correspond to the crack plane are also created so that the orientation of the nodes in the face meet the requirementcounterclockwise for nodes in the above surface and clockwise orientation for the nodes in the below surface preserve the correct orientation of the partitioned tetrahedrons. The centroid of the nodes in the face maps is evaluated and the orientation of the new point (node) that belong to the above face map is set to +100 , and -100 for the point (node) below the face map. The centroid of the two volumes is evaluated with orientation +1000 for the node that belongs to the volume above the crack and orientation set to -1000 for the node that belongs to the volume below the 


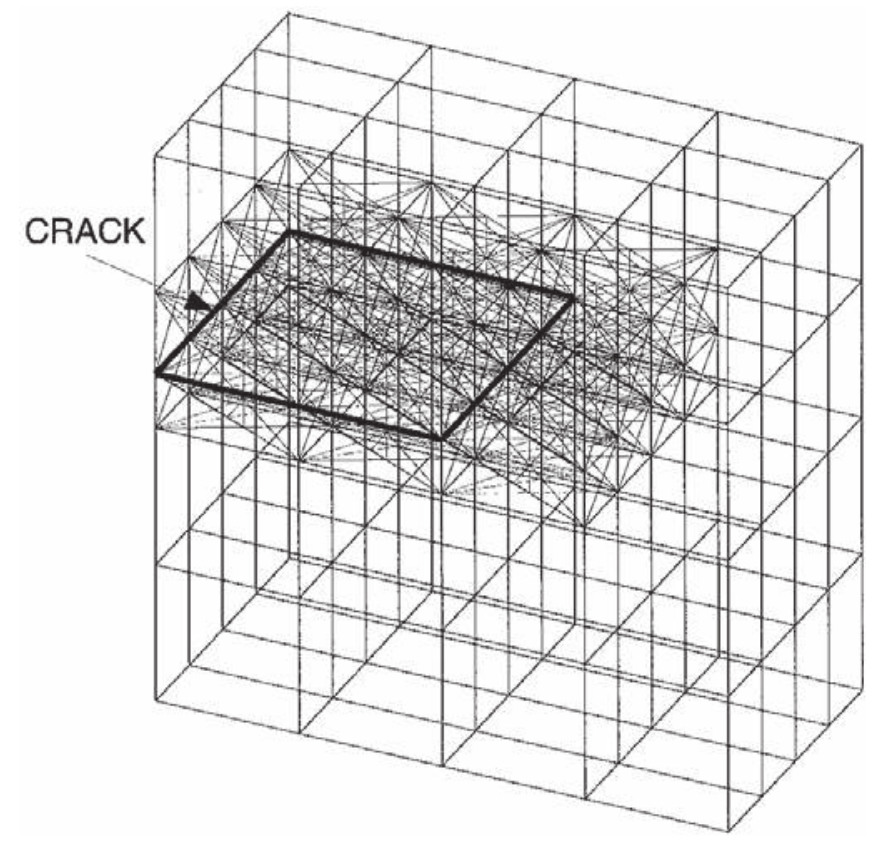

Figure 3. Partitioning algorithm for an edge crack in three dimensions.

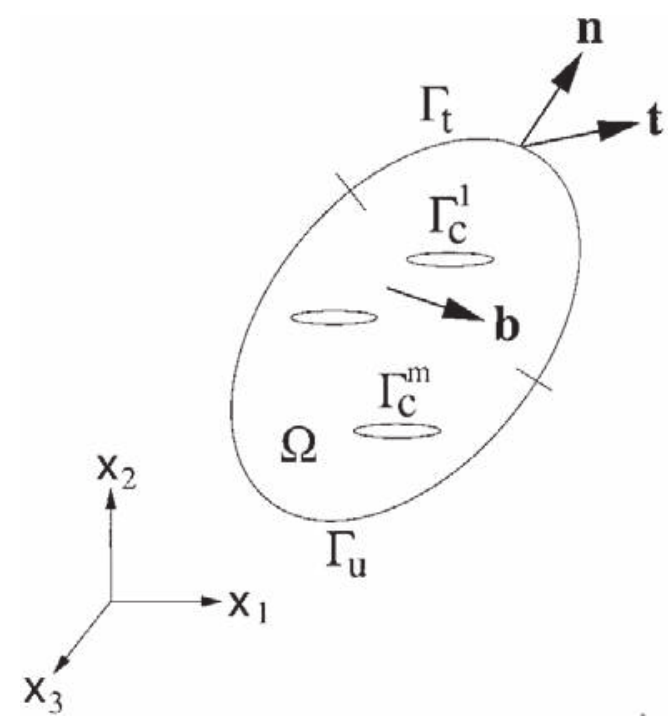

Figure 4. Elastostatic boundary value problem.

crack. A loop over the two face maps is carried out and using the centroid for each face and the centroid of the two volumes, the partitioned elements are obtained. The partitioned elements for an edge crack in a $4 \times 4 \times 4$ hexahedral mesh are shown in Figure 3 .

4.1.4. Nodal enrichment. We next describe the enrichment for crack modelling. The enriched finite element approximation is

$$
\mathbf{u}^{h}(\mathbf{x})=\sum_{\substack{I \\ n_{I} \in \mathbf{N}}} \phi_{I}(\mathbf{x}) \mathbf{u}_{I}+\sum_{\substack{J \\ n_{J} \in \mathbf{N}^{\mathrm{c}}}} \phi_{J}(\mathbf{x}) H(\mathbf{x}) \mathbf{a}_{J}+\sum_{\substack{K \\ n_{K} \in \mathbf{N}^{\mathrm{f}}}} \phi_{K}(\mathbf{x})\left(\sum_{l=1}^{4} \psi_{l}(\mathbf{x}) \mathbf{b}_{K}^{l}\right)
$$

The second and third terms on the right-hand side of the above equation are the discontinuity and front enrichments, respectively. The set $\mathbf{N}^{f}$ consists of those nodes for which the closure of the nodal shape function support intersects the crack front. The set $\mathbf{N}^{c}$ is the set of nodes whose nodal shape function support is intersected by the crack and which do not belong to $\mathbf{N}^{\mathrm{f}}$ :

$$
\begin{aligned}
& \mathbf{N}^{\mathrm{f}}=\left\{n_{K}: n_{K} \in \mathbf{N}, \bar{\omega}_{K} \cap \Lambda_{\mathrm{c}} \neq \emptyset\right\} \\
& \mathbf{N}^{\mathrm{c}}=\left\{n_{J}: n_{J} \in \mathbf{N}, \omega_{J} \cap \Gamma_{\mathrm{c}} \neq \emptyset, n_{J} \notin \mathbf{N}^{\mathrm{f}}\right\}
\end{aligned}
$$

Note that for any node in $\mathbf{N}^{c}$, the support of the nodal shape function is fully cut into two disjoint pieces by the crack. If for a certain node $n_{I}$, one of the two pieces is very small compared to the other, then the generalized Heaviside function used for the enrichment is almost a constant over the support, leading to an ill-conditioned stiffness matrix. Therefore, in this case, node $n_{I}$ is removed from the set $\mathbf{N}^{c}$. The criterion for nodal inclusion in $\mathbf{N}^{\mathrm{c}}$ is as follows: the volume above the crack is $V_{\text {above }}$, and the volume below the crack is $V_{\omega}^{\text {below }}: V_{\omega}=V_{\omega}^{\text {above }}+V_{\omega}^{\text {below }}$. If either of the 
two ratios, $V_{\omega}^{\text {above }} / V_{\omega}$ or $V_{\omega}^{\text {below }} / V_{\omega}$ is below a prescribed tolerance, the node is removed from the set $N^{\mathrm{c}}$. We use a tolerance $\varepsilon=10^{-4}$.

\section{GOVERNING EQUATIONS}

\subsection{Strong form}

Consider a body $\Omega \subset \mathbb{R}^{3}$, with boundary $\Gamma$. The boundary $\Gamma$ consists of the sets $\Gamma_{u}, \Gamma_{\mathrm{t}}$, and $\Gamma_{\mathrm{c}}^{i}$, such that $\Gamma=\Gamma_{u} \cup \Gamma_{\mathrm{t}} \cup_{i=1}^{m} \Gamma_{\mathrm{c}}^{i}$. All the internal surfaces $\Gamma_{\mathrm{c}}^{i}$ are assumed to be traction-free. The boundary value problem of elastostatics solves for the displacement $\mathbf{u}(\mathbf{x})$ of a body $\Omega$ which is fixed on $\Gamma_{u}$ and subjected to surface forces (tractions) along $\Gamma_{\mathrm{t}}$ (Figure 4). We now consider the boundary value problem for small displacement elastostatics. The field equations of elastostatics are

$$
\begin{aligned}
\boldsymbol{\nabla} \cdot \boldsymbol{\sigma}+\mathbf{b} & =0 \quad \text { in } \Omega \\
\boldsymbol{\sigma} & =\mathbf{C}: \boldsymbol{\varepsilon} \\
\boldsymbol{\varepsilon} & =\nabla_{s} \mathbf{u}
\end{aligned}
$$

where $\nabla_{s}$ is the symmetric gradient operator and $\mathbf{C}$ is the tensor of elastic moduli for a homogeneous isotropic material.

The essential and natural boundary conditions are

$$
\begin{aligned}
& \mathbf{u}=\overline{\mathbf{u}} \quad \text { on } \Gamma_{u} \\
& \boldsymbol{\sigma} \cdot \mathbf{n}=\overline{\mathbf{t}} \quad \text { on } \Gamma_{\mathrm{t}} \\
& \boldsymbol{\sigma} \cdot \mathbf{n}=0 \quad \text { on } \Gamma_{\mathrm{c}}^{i} \quad(i=1,2, \ldots, m)
\end{aligned}
$$

where $\mathbf{n}$ is the unit outward normal to $\Omega, \overline{\mathbf{u}}$ and $\overline{\mathbf{t}}$ are prescribed displacements and tractions, respectively, and $m$ is the number of internal surfaces. Note that Equation (10c) imposes the condition that the internal surfaces $\Gamma_{\mathrm{c}}^{i}$ be traction-free.

\subsection{Weak form and discrete system}

We consider the weak form for the equilibrium equation of elasticity with the associated boundary conditions. For the discrete system, the weak form (principle of virtual work) is

$$
\begin{aligned}
& \text { Find } \mathbf{u}^{h} \in \mathbf{V}^{h} \text { such that } \\
& \int_{\Omega^{h}} \boldsymbol{\sigma}\left(\mathbf{u}^{h}\right): \boldsymbol{\varepsilon}\left(\mathbf{v}^{h}\right)=\int_{\Omega^{h}} \mathbf{b} \cdot \mathbf{v} \mathrm{d} \Omega+\int_{\Gamma_{\mathrm{t}}^{h}} \overline{\mathbf{t}} \cdot \mathbf{v} \mathrm{d} \Gamma \quad \forall \mathbf{v}^{h} \in \mathbf{V}_{0}^{h}
\end{aligned}
$$

where $\mathbf{u}^{h}(\mathbf{x}) \in \mathbf{V}^{h}$ and $\mathbf{v}^{h}(\mathbf{x}) \in \mathbf{V}_{0}^{h}$ are the approximating trial and test functions used in X-FEM. Since we are not concerned with convergence proofs, we restrict the discussion to those spaces used in the construction of the discrete approximation. The space $\mathbf{V}^{h}$ is the enriched finite element space that satisfy the Dirichlet boundary conditions, and which include basis functions that are 
discontinuous across the crack surfaces. The space $\mathbf{V}_{0}^{h}$ is the corresponding space with homogeneous Dirichlet boundary conditions.

In a Bubnov-Galerkin procedure, the trial functions $\mathbf{u}^{h}$ as well as the test functions $\mathbf{v}^{h}$ are represented as linear combinations of the same shape functions. The trial and test functions, which are based on Equation (6) are

$$
\begin{aligned}
& \mathbf{u}^{h}(\mathbf{x})=\sum_{\substack{I \\
n_{I} \in \mathbf{N}}} \phi_{I}(\mathbf{x}) \mathbf{u}_{I}+\sum_{\substack{J \\
n_{J} \in \mathbf{N}^{\mathrm{c}}}} \phi_{J}(\mathbf{x}) H(\mathbf{x}) \mathbf{a}_{J}+\sum_{\substack{K \\
n_{K} \in \mathbf{N}^{\mathrm{f}}}} \phi_{K}(\mathbf{x})\left(\sum_{l=1}^{4} \psi_{l}(\mathbf{x}) \mathbf{b}_{K}^{l}\right) \\
& \mathbf{v}^{h}(\mathbf{x})=\sum_{\substack{I \\
n_{I} \in \mathbf{N}}} \phi_{I}(\mathbf{x}) \mathbf{v}_{I}+\sum_{\substack{J \\
n_{J} \in \mathbf{N}^{\mathrm{c}}}} \phi_{J}(\mathbf{x}) H(\mathbf{x}) \mathbf{c}_{J}+\sum_{\substack{K \\
n_{K} \in \mathbf{N}^{\mathrm{f}}}} \phi_{K}(\mathbf{x})\left(\sum_{l=1}^{4} \psi_{l}(\mathbf{x}) \mathbf{e}_{K}^{l}\right)
\end{aligned}
$$

where $\phi_{I}(\mathbf{x})$ are the finite element shape functions, and $\psi_{j}(\mathbf{x})(j=1-4)$ are the enriched functions for the crack front, which are given in Equation (4).

On substituting the trial and test functions from Equation (12) into Equation (11), and using the arbitrariness of nodal variations, the following discrete system of linear equations is obtained:

$$
\mathbf{K d}=\mathbf{f}
$$

where $\mathbf{d}$ is the vector of unknowns at the nodes, and the stiffness matrix $\mathbf{K}$ is

$$
\begin{aligned}
\mathbf{K}_{I J} & =\left[\begin{array}{lll}
\mathbf{K}_{I J}^{u u} & \mathbf{K}_{I J}^{u a} & \mathbf{K}_{I J}^{u b} \\
\mathbf{K}_{I J}^{a u} & \mathbf{K}_{I J}^{a a} & \mathbf{K}_{I J}^{a b} \\
\mathbf{K}_{I J}^{b u} & \mathbf{K}_{I J}^{b a} & \mathbf{K}_{I J}^{b b}
\end{array}\right] \\
\mathbf{K}_{I J}^{\alpha \beta} & =\int_{\Omega^{h}}\left(\mathbf{B}_{I}^{\alpha}\right)^{\mathrm{T}} \mathbf{C} \mathbf{B}_{J}^{\beta} \mathrm{d} \Omega \quad(\alpha, \beta=u, a, b)
\end{aligned}
$$

In Equation (14), the external force vector $\mathbf{f}$ is defined as

$$
\begin{aligned}
\mathbf{f}_{I} & =\left\{\mathbf{f}_{I}^{u}, \mathbf{f}_{I}^{a}, \mathbf{f}_{I 1}^{b}, \mathbf{f}_{I 2}^{b}, \mathbf{f}_{I 3}^{b}, \mathbf{f}_{I 4}^{b}\right\} \\
\mathbf{f}_{I}^{u} & =\int_{\Gamma_{\mathrm{t}}^{h}} \phi_{I} \overline{\mathbf{t}} \mathrm{d} \Gamma+\int_{\Omega^{h}} \phi_{I} \mathbf{b} \mathrm{d} \Omega \\
\mathbf{f}_{I}^{a} & =\int_{\Gamma_{\mathrm{t}}^{h}} \phi_{I} H \overline{\mathbf{t}} \mathrm{d} \Gamma+\int_{\Omega^{h}} \phi_{I} H \mathbf{b} \mathrm{d} \Omega \\
\mathbf{f}_{I j}^{b} & =\int_{\Gamma_{\mathrm{t}}^{h}} \phi_{I} \psi_{j} \overline{\mathbf{t}} \mathrm{d} \Gamma+\int_{\Omega^{h}} \phi_{I} \psi_{j} \mathbf{b} \mathrm{d} \Omega \quad(j=1-4)
\end{aligned}
$$

The discrete equations have three degrees of freedom for unenriched nodes. Nodes in the set $\mathbf{N}^{\mathrm{c}}$ have each three degrees of freedom, and nodes in the set $\mathbf{N}^{f}$ have each twelve degrees of freedom-see Section 4.1.4 for details on the nodal sets $\mathbf{N}^{\mathrm{c}}$ and $\mathbf{N}^{\mathrm{f}}$. 
In Equation (15), $\mathbf{C}$ is the constitutive matrix for an isotropic linear elastic material, and $\mathbf{B}_{I}^{u}$, $\mathbf{B}_{I}^{a}$, and $\mathbf{B}_{I j}^{b}$ are the matrix of shape function derivatives which are given by

$$
\begin{aligned}
& \mathbf{B}_{I}^{u}=\left[\begin{array}{ccc}
\phi_{I, x} & 0 & 0 \\
0 & \phi_{I, y} & 0 \\
0 & 0 & \phi_{I, z} \\
0 & \phi_{I, z} & \phi_{I, y} \\
\phi_{I, z} & 0 & \phi_{I, x} \\
\phi_{I, y} & \phi_{I, x} & 0
\end{array}\right] \\
& \mathbf{B}_{I}^{a}=\left[\begin{array}{ccc}
\left(\phi_{I} H\right)_{, x} & 0 & 0 \\
0 & \left(\phi_{I} H\right)_{, y} & 0 \\
0 & 0 & \left(\phi_{I} H\right)_{, z} \\
0 & \left(\phi_{I} H\right)_{, z} & \left(\phi_{I} H\right)_{, y} \\
\left(\phi_{I} H\right)_{, z} & 0 & \left(\phi_{I} H\right)_{, x} \\
\left(\phi_{I} H\right)_{, y} & \left(\phi_{I} H\right)_{, x} & 0
\end{array}\right] \\
& \mathbf{B}_{I}^{b}=\left[\begin{array}{llll}
\mathbf{B}_{I 1}^{b} & \mathbf{B}_{I 2}^{b} & \mathbf{B}_{I 3}^{b} & \mathbf{B}_{I 4}^{b}
\end{array}\right] \\
& \mathbf{B}_{I j}^{b}=\left[\begin{array}{ccc}
\left(\phi_{I} \psi_{j}\right)_{, x} & 0 & 0 \\
0 & \left(\phi_{I} \psi_{j}\right)_{, y} & 0 \\
0 & 0 & \left(\phi_{I} \psi_{j}\right)_{, z} \\
0 & \left(\phi_{I} \psi_{j}\right)_{, z} & \left(\phi_{I} \psi_{j}\right)_{, y} \\
\left(\phi_{I} \psi_{j}\right)_{, z} & 0 & \left(\phi_{I} \psi_{j}\right)_{, x} \\
\left(\phi_{I} \psi_{j}\right)_{, y} & \left(\phi_{I} \psi_{j}\right)_{, x} & 0
\end{array}\right] \quad(j=1-4)
\end{aligned}
$$

\section{NUMERICAL RESULTS}

Several problems are presented to illustrate the accuracy and versatility of the extended finite element method in three-dimensional elastostatics. We first solve some benchmark crack problems, and then examine the performance of X-FEM for cracks in finite specimens. Finite element mesh generation is carried out by gmsh [27], which is available in the public-domain. In all problems, numerical integration is carried out using Gauss-Legendre quadrature. In hexahedral elements associated with only the finite element shape functions, $2 \times 2 \times 2$ quadrature is used, and in elements that also have enriched degrees of freedom, $6 \times 6 \times 6$ quadrature is used. In all problems, the generalized Heaviside function $H(\mathbf{x})$ and the two-dimensional asymptotic crack-tip displacement functions (see Section 3) are used to model the crack. A conjugate gradient sparse iterative solver $\mathrm{IML}^{++}[28]$ is used with the convergence tolerance $\varepsilon$ set to $10^{-8}$. The elastic constants used in the computations are: Young's modulus $E=10^{5} \mathrm{psi}$ and Poisson's ratio $v=0.3$. The implementation of the domain form of the contour $J$-integral and the parameters used in the computations are described in the following section. 


\subsection{Computation of stress intensity factors}

Domain integral methods [29-31] are used to evaluate stress intensity factors along the threedimensional crack front. For the mode $I$ crack problems considered here, the stress intensity factor at a point $s$ on the crack front is given by

$$
K_{I}(s)=\sqrt{\frac{J(s) E}{1-v^{2}}}
$$

With the normal to the crack front (and in the crack plane) oriented along the $\hat{x}_{1}$-axis of a local co-ordinate system, the pointwise $J$-integral is given by

$$
J(s)=\lim _{\Gamma \rightarrow 0} \int_{\Gamma(s)} H_{\hat{1} \beta} n_{\beta} \mathrm{d} \Gamma \quad(\beta=\hat{1}, \hat{2})
$$

where

$$
H_{\hat{1} j}=W \delta_{\hat{1} j}-\sigma_{i j} u_{i, \hat{1}} \quad(i, j=\hat{1}, \hat{2}, \hat{3})
$$

Domain integral representations of the crack-tip contour integral provide a convenient and accurate method for evaluating stress intensity factors in two- or three-dimensional fracture. For linear elastostatics, in the absence of body forces and material inhomogeneities, and assuming tractionfree crack surfaces, the volume form of the domain integral is given by [29]

$$
J(s)=-\frac{\int_{V}\left(H_{k j} q_{k, j}+H_{k j, j} q_{k}\right) \mathrm{d} V}{\int_{L_{\mathrm{c}}} l_{k} n_{k} \mathrm{~d} s}
$$

where $V$ is a volume enclosing the crack front, $n_{k}(s)$ are components of the in-plane unit outward normal at $s, l_{k}(s)$ are components of an arbitrary unit vector at $s$ lying in the plane of the crack and $L_{\mathrm{c}}$ is the perturbed segment (virtual extension) along the crack front (Figure 5). The vector field $q_{k}$ is defined in $V$ as

$$
q_{k}=\left\{\begin{array}{cc}
l_{k} & \text { on } S_{\mathrm{t}} \\
0 & \text { on } S_{0} \\
\text { arbitrary } & \text { otherwise }
\end{array}\right.
$$

6.1.1. Implementation in $X$-FEM. The mode $I$ stress intensity factors are computed by the domain form of the contour $J$-integral. Consider a point $s$ on the crack front where the stress intensity factor is to be computed. In order to evaluate Equation (21), a virtual extension domain is required around the point $s$ on the crack front [20]. Typically, in fracture analysis with finite elements, the mesh is constructed so that the virtual extension domain is the union of finite elements in the vicinity of the point $s$. Since the crack is not modelled as part of the finite element mesh in $\mathrm{X}-\mathrm{FEM}$, this is not a natural choice. In the two-dimensional implementation of X-FEM [24], elements that were within a characteristic distance of the crack-tip were included in the virtual extension domain, which does not readily generalize to an easy-to-implement algorithm in three dimensions. An alternative approach is to use an independent grid of hexahedral cells around the point $s$ to define the virtual extension domain. This approach is used in EFG for fracture problems, and we adopt the same approach in the three-dimensional implementation of X-FEM. 


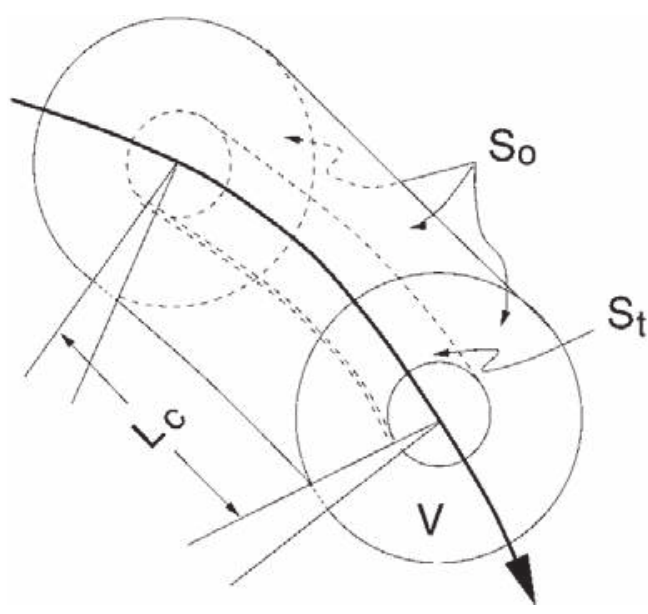

Figure 5. Volume representation for $J$-integral evaluation.

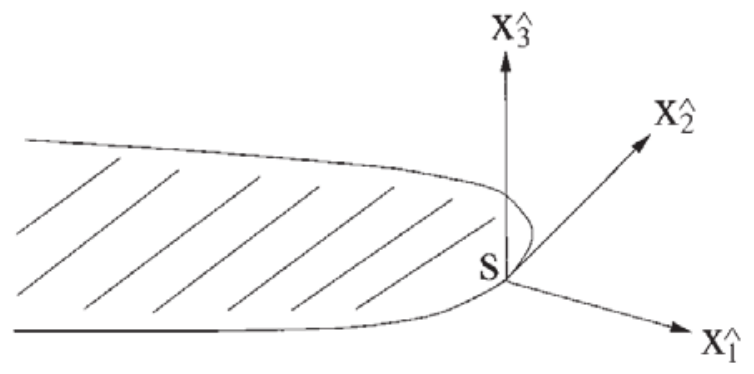

Figure 6. Local crack front co-ordinate axes for virtual extension domain.

We describe the virtual extension domain for the elastostatic problems presented in Section 6. Let the perturbed segment $\left(L_{\mathrm{c}}\right)$ along the crack front be parameterized by the curvilinear co-ordinate $s: \mathbb{R}^{3} \rightarrow\left[0, L_{\mathrm{c}}\right]$. Consider the evaluation of the domain integral at a point $s$ on the crack front. The dimensions of the virtual extension domain are: $L_{\hat{1}}, L_{\hat{2}}$ and $L_{\hat{3}}$ along the $x_{\hat{1}}^{-}, x_{\hat{2}^{-}}$, and $x_{\hat{3}}$-co-ordinate directions, respectively. The point $s$ is located at the origin of the local orthogonal $x_{\hat{1}} x_{\hat{2}} x_{\hat{3}}$-co-ordinate system (Figure 6 ). The $x_{\hat{2}}$-axis is contained in the plane of the crack and is tangential to the crack front at $s$. The $J$-domain is sub-divided into $6 \times 2 \times 6$ hexahedral cells, and $6 \times 6 \times 6$ quadrature rule is used in each integration cell. In three-dimensional fracture, the denominator in Equation (21) is well approximated by the formula $\left(L_{\hat{2}}^{+}+L_{\hat{2}}^{-}\right) / 2$ [32], where $L_{\hat{2}}^{+}$and $L_{\hat{2}}^{-}$are the element lengths on either side of $s$ in the $\hat{x}_{2}$-direction. On using the same approximation, we obtain

$$
\int_{L_{\mathrm{c}}} l_{k} n_{k} \mathrm{~d} s \approx \frac{L_{\hat{2}}}{2}
$$

which is used in the X-FEM domain integral computations. We define the vector function $q_{k}$ in the local crack-front co-ordinate system. The function $q_{\hat{1}}$ is chosen to be a trilinear function which vanishes on the boundary of the virtual extension domain and is unity at the point $s$. In the local crack-front co-ordinate system,

$$
q_{\hat{1}}\left(x_{\hat{1}}, x_{\hat{2}}, x_{\hat{3}}\right)=\left(1-\frac{2\left|x_{\hat{1}}\right|}{L_{\hat{1}}}\right)\left(1-\frac{2\left|x_{\hat{\hat{2}}}\right|}{L_{\hat{2}}}\right)\left(1-\frac{2\left|x_{\hat{3}}\right|}{L_{\hat{3}}}\right), \quad q_{\hat{2}}=0, \quad q_{\hat{3}}=0
$$

with the aid of the above formulas for various terms that appear in Equation (21), we evaluate the domain form of the $J$-integral.

\subsection{Benchmark problems}

For an infinite domain, two well known and widely used benchmarks are the penny crack and the elliptical crack under pure mode $I$ loading conditions. The problem of an edge crack in a finitethickness plate under tension [33] is also used to study the accuracy of new numerical techniques in three-dimensional SIF computations. We compare the numerical stress intensity factors to the SIF solutions for the above problems. 


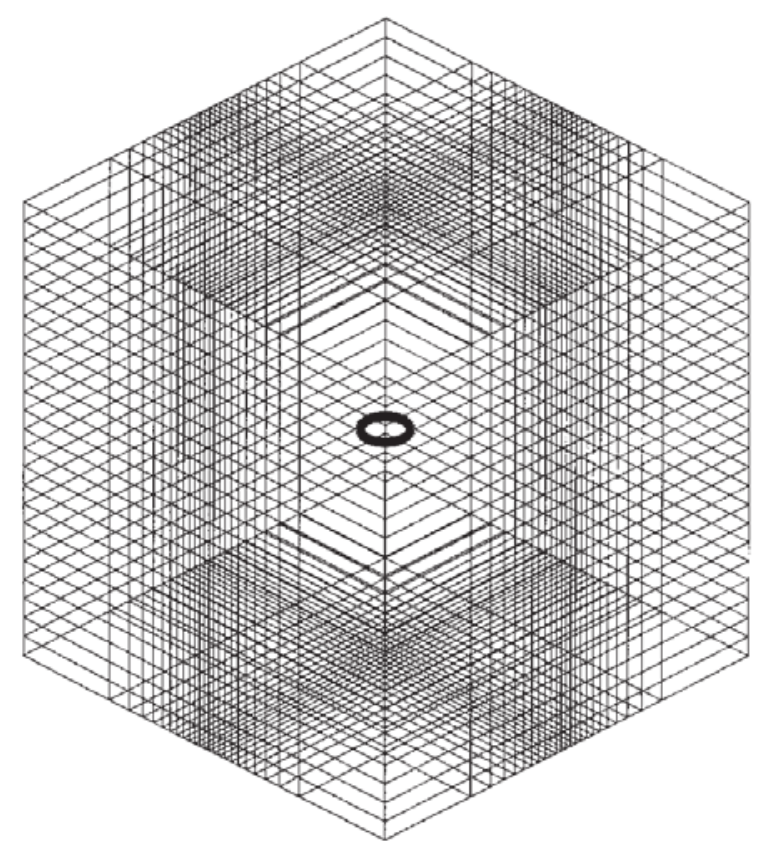

Figure 7. Mesh (surface) for the penny crack problem.

6.2.1. Planar penny crack in an infinite domain. Let $a$ be the radius of a penny crack with $x_{3}$ the co-ordinate axis normal to the plane of the crack. The closed-form solution for the stress intensity factor along the crack front of a penny crack in an infinite domain under uniaxial tension is given by $[34,35]$

$$
K_{I}^{\mathrm{E}}=2 \sigma_{33}^{0} \sqrt{\frac{a}{\pi}}
$$

Consider a penny crack of radius $a=0.1$ inside a bi-unit cube. The specimen is subjected to the stress $\sigma_{33}^{0}=1$ in the $x_{3}$-direction. Since the effects of the finite size of the model are minimal, we use the exact solution given in Equation (25). Two different meshes are used: (a) Mesh 1 consists of $24 \times 24 \times 24$ hexahedral elements, and (b) Mesh 2 has $24 \times 24 \times 25$ hexahedral elements. In the former, the crack lies on element faces, whereas in the latter, the crack is located in the centre of the elements (no intersection with any element faces). Both meshes have graded refinement towards the centre of the cube and in the plane of the crack (Figure 7). The nodes normal to the crack surface are equi-spaced with length $h$.

In Figure 8, a contour plot of the crack plane $\left(x_{3}=0\right)$ nodal enrichment for the $24 \times 24 \times 24$ mesh is shown. The domain of enrichment extends an element length $(h)$ above and below the crack plane: identical contour plots to that shown appear on $x_{3}= \pm h$. In Figure 8(a), the nodes enriched on the crack plane by the generalized Heaviside function are indicated, and in Figure $8(\mathrm{~b})$, the nodes enriched by the crack-tip functions are shown. In both the contour plots, colour codes are assigned a number between 0 and 4, where the number indicates the number of nodes on the face of the element that are enriched. As one can observe, the mesh is fairly coarse in the vicinity of the crack front. In Table I, the parameters for the system equations are indicated.

The dimensions of the virtual extension domain are: $L_{\hat{1}}=2 a, L_{\hat{2}}=a / 2, L_{\hat{3}}=2 a$, and $L_{\hat{1}}=L_{\hat{2}}=$ $a / 2, L_{\hat{3}}=2.5 a$ for meshes 1 and 2 , respectively. The SIF results have four-fold symmetry; hence results for only $0^{\circ} \leqslant \theta \leqslant 90^{\circ}$ are presented. In Table II, the numerical SIF results are presented as a function of $\theta$, and in Figure 9, the normalized SIF is shown as a function of $\theta$. The X-FEM 


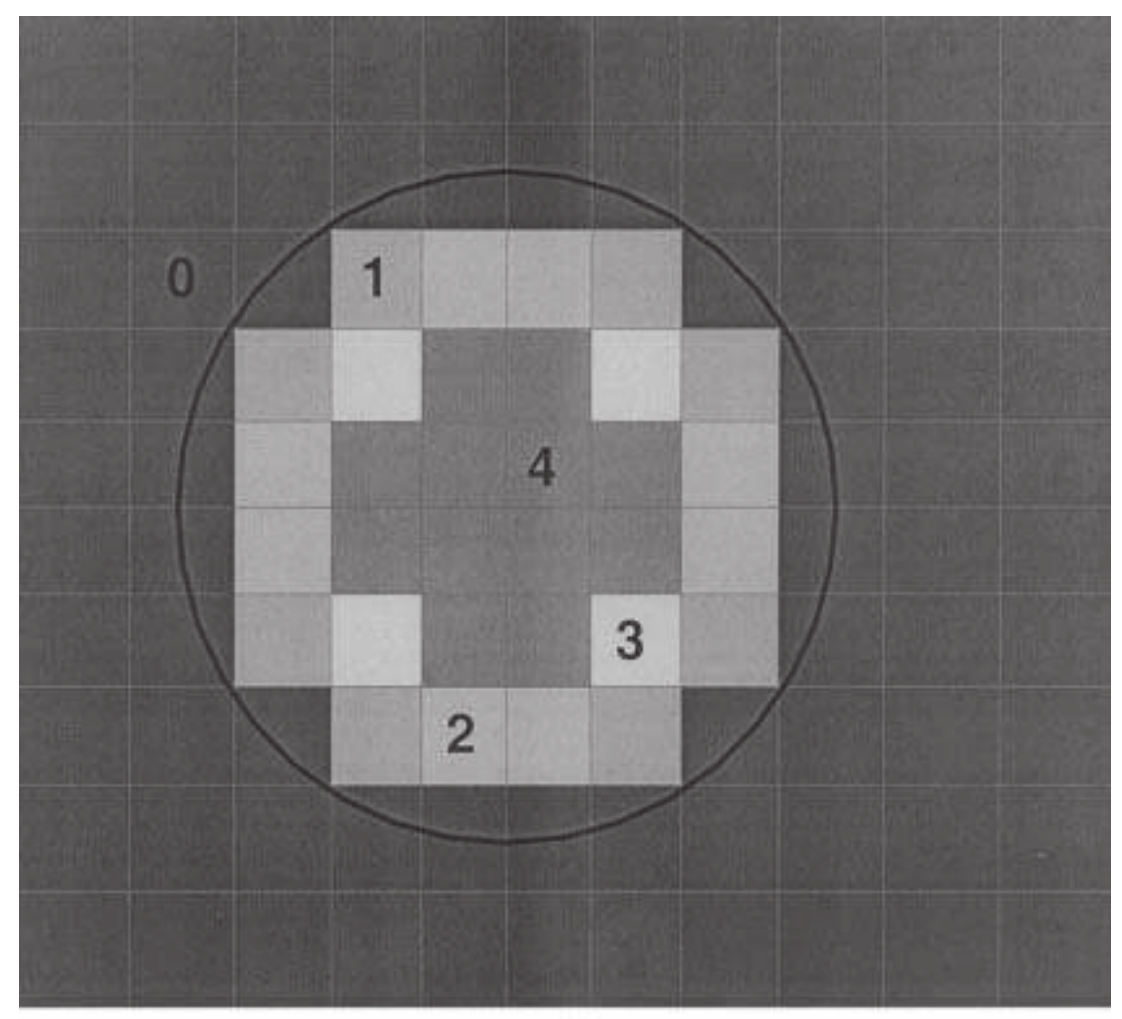

(a)

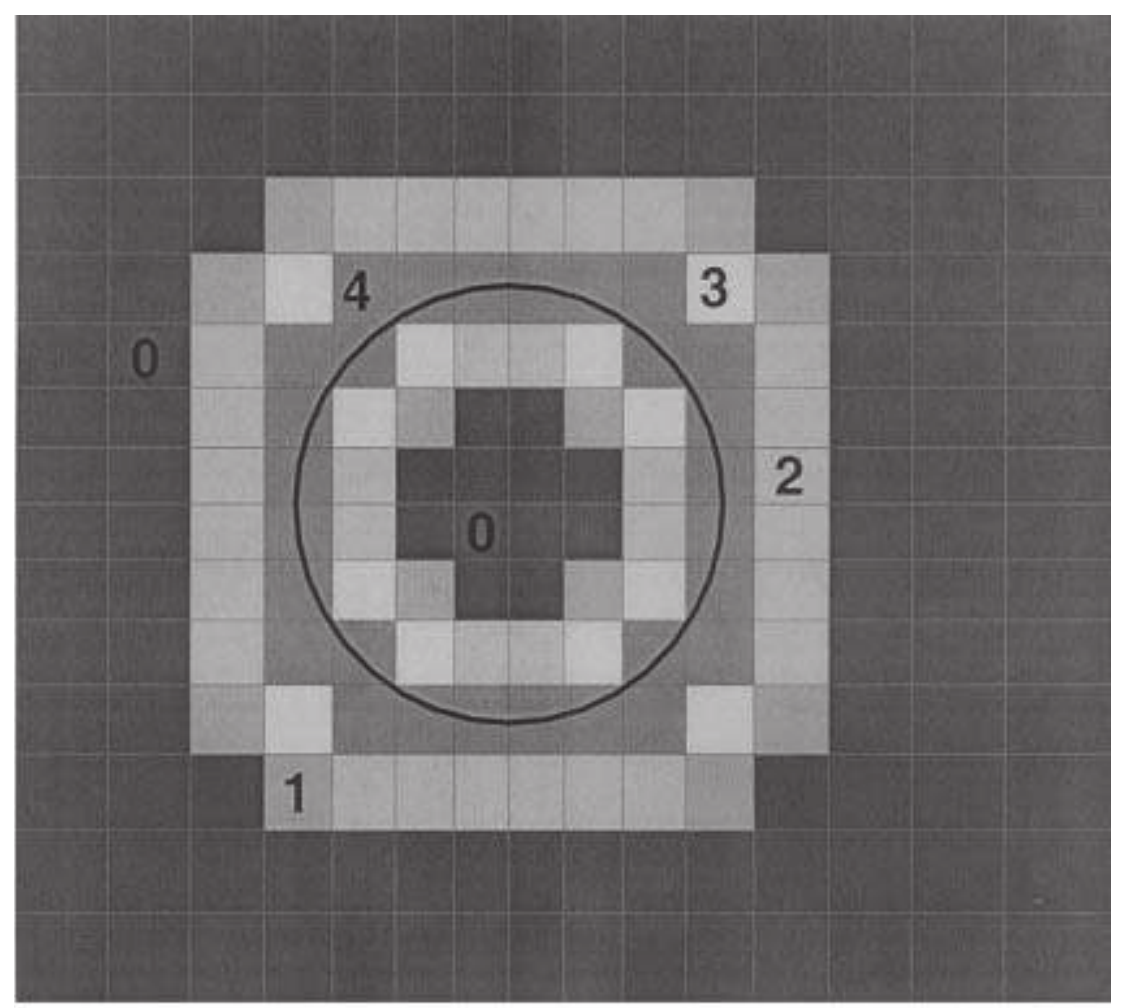

(b)

Figure 8. Nodal enrichment on the crack plane for the penny crack problem: (a) generalized Heaviside function; and (b) crack-tip functions. 
Table I. System parameters for the penny crack problem.

\begin{tabular}{lccc}
\hline Mesh & $\begin{array}{c}\text { Number of } \\
\text { unknowns }\end{array}$ & $\begin{array}{c}\text { Non-zero } \\
\text { entries }\end{array}$ & $\begin{array}{c}\text { Sparsity } \\
(\%)\end{array}$ \\
\hline $24 \times 24 \times 24$ & 48948 & 4212194 & 0.17 \\
$24 \times 24 \times 25$ & 42678 & 3531390 & 0.19 \\
\hline
\end{tabular}

Table II. Stress intensity factors for the penny crack problem.

\begin{tabular}{lccc}
\hline $\begin{array}{l}\theta \\
(\mathrm{deg})\end{array}$ & $K_{I}^{\mathrm{E}}$ & $\begin{array}{c}K_{I} \\
{[24 \times 24 \times 24]}\end{array}$ & $\begin{array}{c}K_{I} \\
{[24 \times 24 \times 25]}\end{array}$ \\
\hline 0 & 0.3568 & 0.3508 & 0.3617 \\
10 & 0.3568 & 0.3583 & 0.3503 \\
20 & 0.3568 & 0.3592 & 0.3559 \\
30 & 0.3568 & 0.3467 & 0.3595 \\
40 & 0.3568 & 0.3535 & 0.3383 \\
50 & 0.3568 & 0.3535 & 0.3383 \\
60 & 0.3568 & 0.3467 & 0.3595 \\
70 & 0.3568 & 0.3592 & 0.3559 \\
80 & 0.3568 & 0.3583 & 0.3503 \\
90 & 0.3568 & 0.3508 & 0.3617 \\
\hline
\end{tabular}

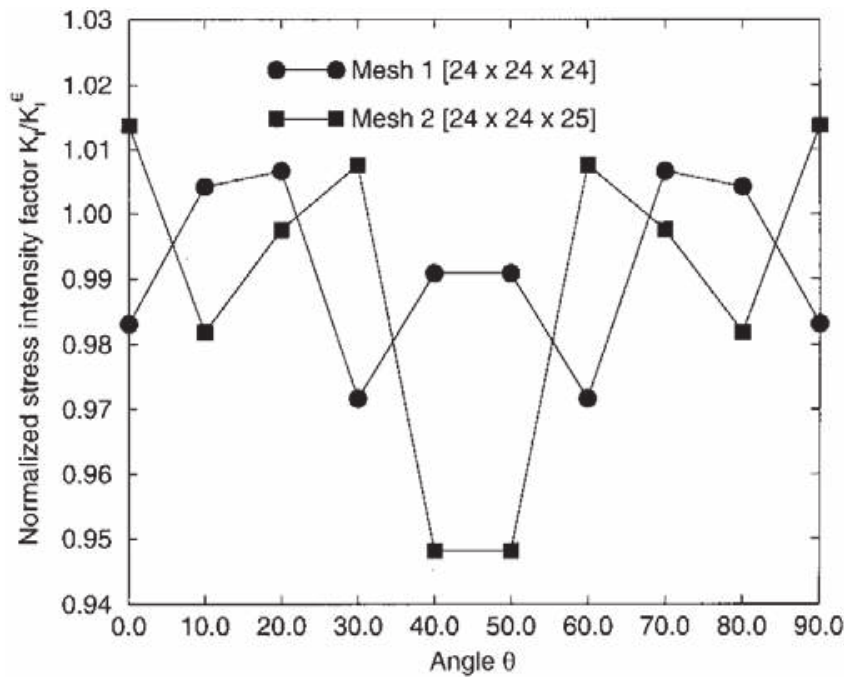

Figure 9. Normalized stress intensity factors for the penny crack problem.

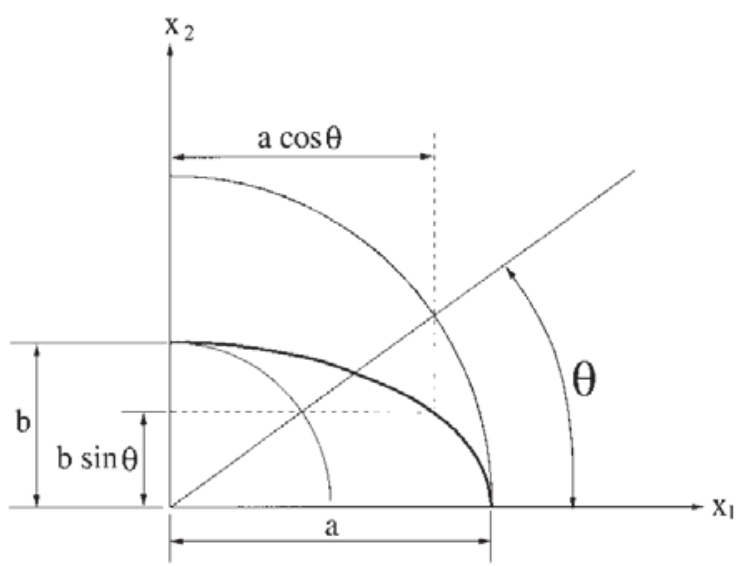

Figure 10. Geometric definitions for an elliptical crack.

results are observed to be in good agreement with the exact solution: the errors in the SIFs are between 0.4-2.9 per cent for mesh 1 and 0.3-5.2 per cent for mesh 2 .

6.2.2. Planar elliptical crack in an infinite domain. We consider an elliptical crack with semimajor axis $a=0.1$ and semi-minor axis $b=0.05$ inside a bi-unit cube. The body is subjected to unit tractions $\sigma_{33}^{0}=1$ on $x_{3}= \pm 1$. The co-ordinate $x_{3}$-axis is normal to the plane of the crack. Since the 
Table III. Stress intensity factors for the elliptical crack problem.

\begin{tabular}{lcc}
\hline $\begin{array}{l}\theta \\
(\mathrm{deg})\end{array}$ & $K_{I}^{\mathrm{E}}$ & $K_{I}$ \\
\hline 0 & 0.2314 & 0.2358 \\
10 & 0.2365 & 0.2378 \\
20 & 0.2495 & 0.2459 \\
30 & 0.2662 & 0.2564 \\
40 & 0.2830 & 0.2776 \\
50 & 0.2983 & 0.2965 \\
60 & 0.3107 & 0.3089 \\
70 & 0.3198 & 0.3163 \\
80 & 0.3253 & 0.3194 \\
90 & 0.3273 & 0.3202 \\
\hline
\end{tabular}

crack dimensions are small compared to the specimen, we use the infinite domain solution as the reference solution. The exact SIF solution for a planar elliptical crack in an infinite domain is [35]

$$
K_{I}^{\mathrm{E}}=\frac{\sigma_{33}^{0} \sqrt{\pi b}}{E(k)}\left\{\sin ^{2} \theta+\frac{b^{2}}{a^{2}} \cos ^{2} \theta\right\}^{1 / 4}
$$

where $\theta$ is the elliptic angle (Figure 10), $\sigma_{33}^{0}$ is the far-field applied stress in the $x_{3}$-direction, and $E(k)$ which is the elliptic integral of the second kind is given by

$$
E(k)=\int_{0}^{\pi / 2} \sqrt{1-k^{2} \sin ^{2} \theta} \mathrm{d} \theta, \quad k^{2}=\frac{a^{2}-b^{2}}{a^{2}}
$$

The finite element mesh consists of $24 \times 24 \times 24$ hexahedral elements. The number of unknowns in the matrix system is 48324 , with 3993602 non-zero entries in the stiffness matrix-matrix sparsity is 0.17 per cent. The dimensions of the virtual extension domain are: $L_{\hat{1}}=2 b, L_{\hat{2}}=b, L_{\hat{3}}=4 b$. The SIF results have four-fold symmetry; hence results for only $0^{\circ} \leqslant \theta \leqslant 90^{\circ}$ are presented. For the chosen values of $a$ and $b$, the value of the elliptic integral $E(k)=1.211096$ [36]. In Table III, the SIF results are presented as a function of the elliptic angle $\theta$, and in Figure 11, the normalized SIF is plotted versus $\theta$. The agreement between the exact solution and the numerical results is good for the entire range of $\theta$. The minimum $\left(\theta=10^{\circ}\right)$ and maximum $\left(\theta=30^{\circ}\right)$ errors in the stress intensity factors are 0.6 and 3.7 per cent, respectively.

6.2.3. Single edge-crack tension specimen. A single edge-crack tension specimen (Figure 12) subjected to unit tractions in the $x_{3}$-direction is analysed. The dimensions of the specimen are: $h / w=1.75, a / w=1.0$, and $t / w=3.0$. This benchmark problem was studied by Raju and Newman [33] using singular finite elements, and by Li et al. [37], who used a symmetric weak-form boundary integral equation method. Two different finite element meshes are considered in the XFEM model: (a) Mesh 1 consists of $20 \times 20 \times 20$ hexahedral elements, with each element a cuboid of dimensions $h_{1} \times h_{2} \times h_{3}\left(h_{1}=0.5, h_{2}=0.75, h_{3}=0.875\right)$; and (b) Mesh 2 has $40 \times 40 \times 40$ hexahedral elements, with each element a cuboid of dimensions $h_{1} \times h_{2} \times h_{3}\left(h_{1}=0.25, h_{2}=0.375\right.$, $\left.h_{3}=0.4375\right)$. Unit tractions $\sigma_{33}^{0}=1$ are imposed on the top and bottom surfaces. The solution 


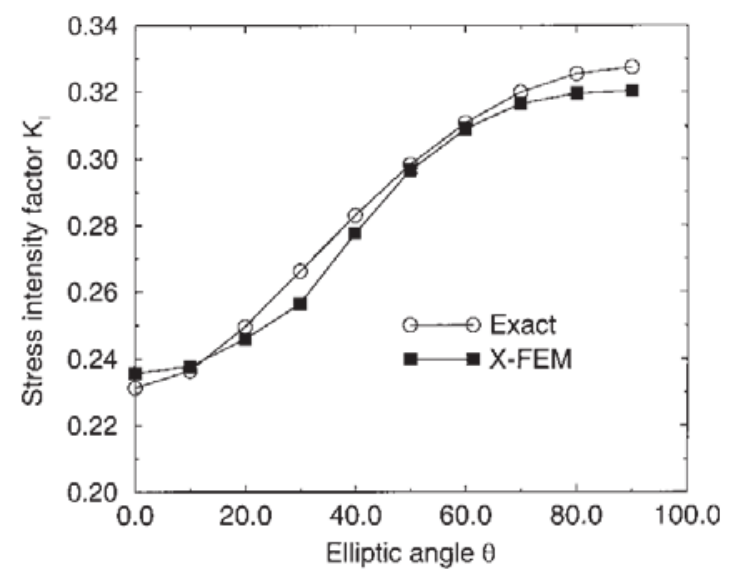

Figure 11. Stress intensity factors for the elliptical crack problem

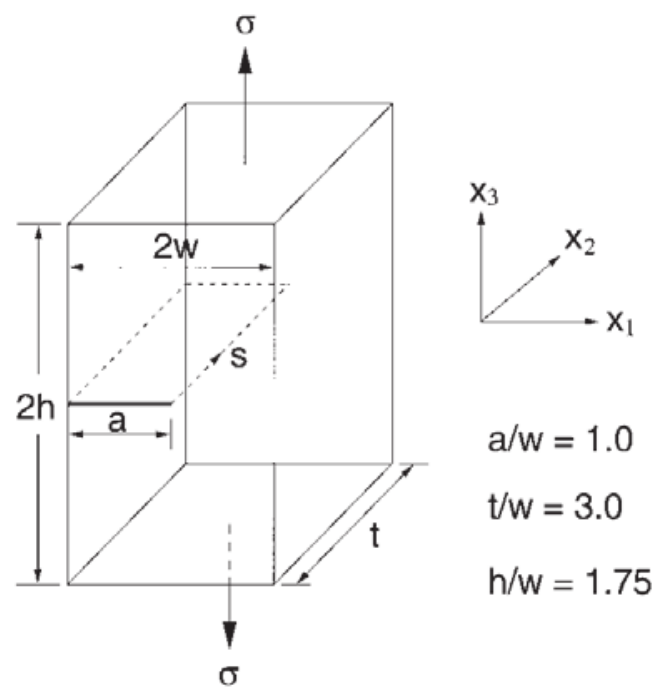

Figure 12. Single edge-crack tension specimen.

Table IV. System parameters for the single edge-crack problem.

\begin{tabular}{lccc}
\hline Mesh & $\begin{array}{c}\text { Number of } \\
\text { unknowns }\end{array}$ & $\begin{array}{c}\text { Non-zero } \\
\text { entries }\end{array}$ & $\begin{array}{c}\text { Sparsity } \\
(\%)\end{array}$ \\
\hline $20 \times 20 \times 20$ & 30612 & 2941268 & 0.30 \\
$40 \times 40 \times 40$ & 213522 & 17955158 & 0.04 \\
\hline
\end{tabular}

parameters for the different meshes are indicated in Table IV. The dimensions of the virtual extension domain are: $L_{\hat{1}}=6 h_{1}, L_{\hat{2}}=2 h_{2}$, and $L_{\hat{3}}=6 h_{2}$.

The SIF results for the hexahedral meshes are presented in Table V, and in Figure 13, comparisons with the results obtained by Raju and Newman [33] and Li et al. [37] are shown. The arc length co-ordinate $s$ is measured along the crack front, from its centre $(s=0)$ to the point where it meets the free surface $(s=t / 2)$. The stress state at the centre of the specimen is under near plane strain conditions and the numerical SIF approaches the two-dimensional plane strain result. Good agreement between the X-FEM results and the reference solutions are obtained in the interior, away from the free surface. The SIF results due to Li et al. [37] are greater than the other numerical results towards the centre of the crack front.

The stress state at the intersection of a crack with a free surface has been the subject of many studies. The order of the singularity at the intersection of a crack and a free surface is $r^{-\lambda}\left(\lambda<\frac{1}{2}\right)$, where $\lambda$ is dependent on the Poisson's ratio [38]. Bazant and Estenssoro [39] investigated the stress singularity index by a special finite element method for mode I-III cracks. Tan and Fenner [40] used boundary integral equations to study the nature of the singularity at the intersection between a crack and a free surface and showed numerically that the stresses (strains) near the free surface are less singular than the inverse square root form, and the effect becomes more pronounced with increasing Poisson's ratio. The trend towards a diminishing $K_{\mathrm{I}}$ as the free surface is approached is observed in the X-FEM as well as other reference solution results. 
Table V. Stress intensity factors for the single edge-crack under uniaxial tension problem.

\begin{tabular}{lcc}
\hline & $\frac{K_{I}}{\sigma_{33}^{0} \sqrt{\pi a}}$ & $\frac{K_{I}}{\sigma_{33}^{0} \sqrt{\pi a}}$ \\
$\frac{s}{t}$ & {$[20 \times 20 \times 20]$} & {$[40 \times 40 \times 40]$} \\
\hline 0.00 & 2.8470 & 2.7873 \\
0.05 & 2.8481 & 2.7869 \\
0.10 & 2.84703 & 2.7858 \\
0.15 & 2.8449 & 2.7836 \\
0.20 & 2.8412 & 2.7799 \\
0.25 & 2.8350 & 2.7736 \\
0.30 & 2.8244 & 2.7629 \\
0.35 & 2.8056 & 2.7441 \\
0.40 & 2.7702 & 2.7084 \\
0.45 & 2.6796 & 2.6283 \\
\hline
\end{tabular}

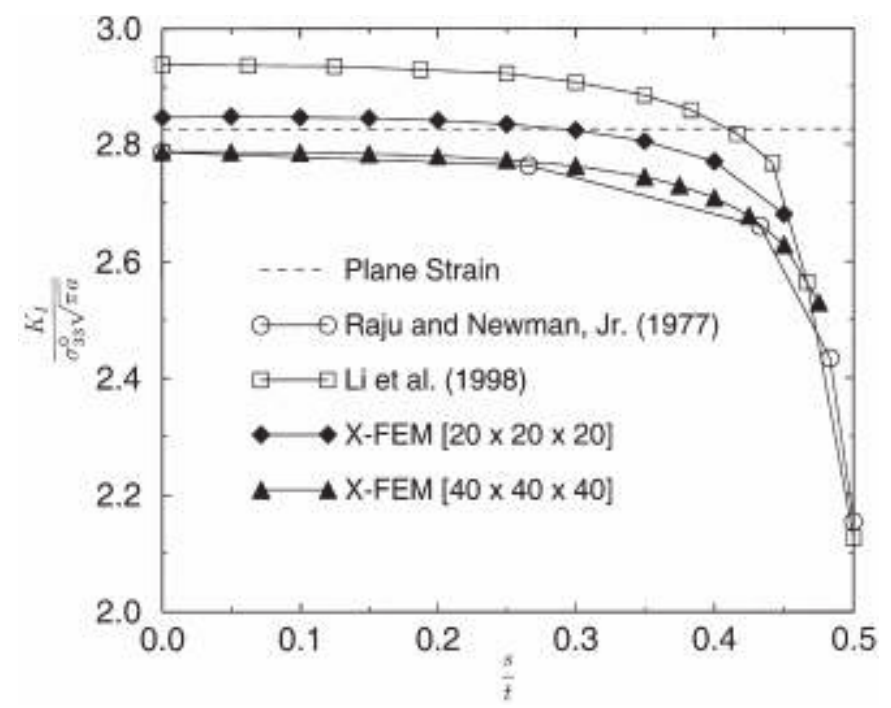

Figure 13. Comparison of stress intensity factors for the single edge-crack problem.

Table VI. System parameters for the penny crack in a finite cube problem.

\begin{tabular}{lccc}
\hline Mesh & $\begin{array}{c}\text { Number of } \\
\text { unknowns }\end{array}$ & $\begin{array}{c}\text { Non-zero } \\
\text { entries }\end{array}$ & $\begin{array}{c}\text { Sparsity } \\
(\%)\end{array}$ \\
\hline $20 \times 20 \times 20$ & 50568 & 4770050 & 0.19 \\
$40 \times 40 \times 40$ & 213252 & 18115070 & 0.04 \\
\hline
\end{tabular}

\subsection{Planar penny crack in a finite cube}

As an example of an embedded crack in a finite domain, we consider the problem of a penny crack of radius $a$ located at the centre of a bi-unit cube. We choose $a=0.5$, and compare the 
Table VII. Stress intensity factors for the penny crack in a finite cube problem.

\begin{tabular}{lcc}
\hline $\begin{array}{l}\theta \\
(\mathrm{deg})\end{array}$ & $\begin{array}{c}K_{I} \\
{[24 \times 24 \times 24]}\end{array}$ & $\begin{array}{c}K_{I} \\
{[40 \times 40 \times 40]}\end{array}$ \\
\hline 0 & 0.8641 & 0.8685 \\
10 & 0.8706 & 0.8792 \\
20 & 0.8848 & 0.8933 \\
30 & 0.8702 & 0.8863 \\
40 & 0.8754 & 0.8920 \\
50 & 0.8754 & 0.8920 \\
60 & 0.8704 & 0.8864 \\
70 & 0.8853 & 0.8935 \\
80 & 0.8709 & 0.8799 \\
90 & 0.8644 & 0.8689 \\
\hline
\end{tabular}

numerical results to those obtained by Li et al. [37] who used a symmetric weak-form boundary integral equation formulation. As a reference solution, a refined mesh solution was adopted in Reference [37] for the stress intensity factor at $\theta=0^{\circ}$ :

$$
K_{I}^{\mathrm{ref}}=\frac{2.213 \sigma_{33}^{0}}{\pi} \sqrt{\pi a}, \quad\left(\theta=0^{\circ}\right)
$$

where $\sigma_{33}^{0}$ is the applied stress in the $x_{3}$-direction. Two different finite element meshes are considered: (a) Mesh 1 consists of $24 \times 24 \times 24$ hexahedral elements, with each element a cube of edge length $h=\frac{1}{12}$; and (b) Mesh 2 has $40 \times 40 \times 40$ hexahedral elements, with each element a cube of edge length $h=0.05$. Unit tractions $\sigma_{33}^{0}=1$ are imposed on the surfaces $x_{3}= \pm 1$. In Table VI, the solution parameters are indicated. The dimensions of the virtual extension domain are: $L_{\hat{1}}=2 h, L_{\hat{2}}=2 h, L_{\hat{3}}=2 h$. In Table VII, the SIF results are presented as a function of $\theta$. With refinement, improved SIFs are obtained for all $\theta$, which indicates the correct trend towards convergence. Comparing the numerical solution at $\theta=0^{\circ}$ to the reference solution given in Equation (28), we observe that the difference between the numerical result and the reference solution for meshes 1 and 2 are about 2.0 per cent and 1.6 per cent, respectively.

\section{CONCLUSIONS}

The formulation and implementation of the extended finite element method (X-FEM) for threedimensional crack modelling was described. In X-FEM, the finite element space is enriched by adding special functions to the approximation using the notion of partition of unity. For threedimensional crack modelling, a discontinuous function was used to model the interior of the crack surface, and functions from the two-dimensional asymptotic crack-tip displacement fields were used for the crack front enrichment. These enrichment functions were added to the finite element approximation within the context of a displacement-based Galerkin formulation.

Issues pertaining to accurate and robust geometric computations in X-FEM were addressed, and some of the algorithms were described. The performance of the extended finite element method for three-dimensional static cracks was studied. Benchmark mode $I$ problems of penny and elliptical 
cracks in an infinite domain were solved. The numerical stress intensity factors (SIFs) were found to be in good agreement with the exact solution for these problems.

The SIFs obtained using X-FEM for a single edge-crack in a finite-thickness specimen approached the plane strain results towards the centre of the specimen and showed a significant decrease as the free surface was approached, which corresponded well to other reference solutions. The SIF results at $\theta=0^{\circ}$ for an embedded penny crack in a finite cube also showed good agreement with the solution due to Li et al. [37].

This study demonstrates the accuracy of the extended finite element method in three-dimensional SIF computations. The method provides a robust and versatile numerical tool to solve crack problems in complex structural components without the need to explicitly align the mesh with the crack. By eliminating the need to include the crack surfaces in the model, mesh generation is greatly simplified. Furthermore, it facilitates the modelling of crack growth for fatigue studies, since a single mesh suffices.

\section{ACKNOWLEDGEMENTS}

The authors are grateful for the research support of the National Science Foundation through contract CMS9732319, the Multi-University Center for Integrated Diagnostics, Georgia Institute of Technology ONR Grant N0014-95-1-0539, the Federal Aviation Administration contract DTFA03-98-F-IA025 and the Office of Naval Research. The authors also thank John Dolbow, Jean-Francois Remacle, and Christophe Geuzaine for many helpful discussions.

\section{REFERENCES}

1. Henshell RD, Shaw KG. Crack tip finite elements are unnecessary. International Journal for Numerical Methods in Engineering 1975; 9:495-507.

2. Barsoum RS. Triangular quarter-point elements as elastic and perfectly-plastic crack tip elements. International Journal for Numerical Methods in Engineering 1977; 11:85-98.

3. Akin JE. The Generation of elements with singularities. International Journal for Numerical Methods in Engineering 1976; 10:1249-1259.

4. Benzley SE. Representation of singularities with isoparametric finite elements. International Journal for Numerical Methods in Engineering 1974; 8:537-545.

5. Gifford Jr LN, Hilton PD. Stress intensity factors by enriched finite elements. Engineering Fracture Mechanics 1978; 10:485-496.

6. Gerstle WH, Martha L, Ingraffea AR. Finite and boundary element modeling of crack propagation in two- and three dimensions. Engineering with Computers 1987; 2:167-183.

7. Gerstle WH, Ingraffea AR, Perucchio R. Three-Dimensional fatigue crack propagation analysis using the boundary element method. International Journal of Fatigue 1988; 10(3):187-192.

8. Martha LF, Wawrzynek PA, Ingraffea AR. Arbitrary crack representation using solid modeling. Engineering with Computers 1993; 9:63-82.

9. Vijayakumar K, Atluri SN. An embedded elliptical flaw in an infinite solid, subject to arbitrary crack-face tractions. Journal of Applied Mechanics 1981; 48:88-96.

10. Nishioka T, Atluri SN. Analytical solution for embedded cracks, and finite element alternating method for elliptical surface cracks, subjected to arbitrary loading. Engineering Fracture Mechanics 1983; 17:247-268.

11. Cruse TA. Boundary Element Analysis in Computational Fracture. Kluwer Academic Publishers: Dordrecht, The Netherlands, 1988.

12. Murakami Y, Nemat-Nasser S. Interacting dissimilar semi-elliptical surface flaws under tension and bending. Engineering Fracture Mechanics 1982; 16(3):373-386.

13. Xu Y, Moran B, Belytschko T. Self-similar crack expansion method for three-dimensional crack analysis. ASME, Journal of Applied Mechanics 1997; 64:729-737.

14. Hills DA, Kelly PA, Dai DN, Korsunsky AM. Solution of Crack Problems: The Distributed Dislocation Technique. Kluwer Academic Publishers: Dordrecht, The Netherlands, 1996.

15. Dai DN, Hills DA, Nowell D. Modelling of growth of three-dimensional cracks by a continuous distribution of dislocation loops. Computational Mechanics 1997; 19:538-544. 
16. Belytschko T, Lu YY, Gu, L. Element-free Galerkin methods. International Journal for Numerical Methods in Engineering 1994; 37:229-256.

17. Fleming M, Chu YA, Moran B, Belytschko T. Enriched element-free Galerkin methods for crack tip fields. International Journal for Numerical Methods in Engineering 1997; 40:1483-1504.

18. Sukumar N, Moran B, Black T, Belytschko T. An element-free Galerkin method for three-dimensional fracture mechanics. Computational Mechanics 1997; 20:170-175.

19. Krysl P, Belytschko T. The element-free Galerkin method for dynamic propagation of arbitrary 3-d cracks. International Journal for Numerical Methods in Engineering 1999; 44(6):767-800.

20. Melenk JM, Babuška I. The partition of unity finite element method: basic theory and applications. Computer Methods in Applied Mechanics and Engineering 1996; 139:289-314.

21. Strouboulis T, Babuška I, Copps K. The design and analysis of the generalized finite element method. Computer Methods in Applied Mechanics and Engineering 2000; 181:43-71.

22. Strouboulis T, Copps K, Babuška I. The generalized finite element method: an example of its implementation and illustration of its performance. International Journal for Numerical Methods in Engineering 2000; 47(8):1401-1417.

23. Belytschko T., Black T. Elastic crack growth in finite elements with minimal remeshing. International Journal for Numerical Methods in Engineering 1999; 45(5):601-620.

24. Moës N, Dolbow J, Belytschko T. A finite element method for crack growth without remeshing. International Journal for Numerical Methods in Engineering 1999; 46(1):131-150.

25. Dolbow J. An extended finite element method with discontinuous enrichment for applied mechanics. Ph.D. Thesis, Theoretical and Applied Mechanics, Northwestern University, Evanston, IL, USA, 1999.

26. Shewchuk JR. Adaptive precision floating-point arithmetic and fast robust geometric predicates. Discrete and Computational Geometry 1997; 18:305-363.

27. Remacle JF, Geuzaine C. Gmsh finite element grid generator. Available at http://scorec_rpi_edu/ remacle/ Gmsh_Eng.html, 1998.

28. Dongarra J, Lumsdaine A, Pozo R, Remington K. IML version 1.2: Iterative methods library reference guide. Technical Report, 1998. Available at http://www.math.nist.gov/iml++.

29. Moran B, Shih CF. Crack tip and associated domain integrals from momentum and energy balance. Engineering Fracture Mechanics 1987; 27(6):615-641.

30. Moran B, Shih C.F. A general treatment of crack tip contour integrals. International Journal of Fracture 1987; 35:295-310.

31. Nikishkov GP, Atluri SN. Calculation of fracture mechanics parameters for an arbitrary three-dimensional crack by the 'equivalent domain integral method'. International Journal for Numerical Methods in Engineering 1987; 24:1801-1821.

32. Gosz M, Dolbow J, Moran B. Domain integral formulation for stress intensity factor computation along curved threedimentional interface cracks. International Journal of Solids and Structures 1998; 35:1763-1783.

33. Raju IS, Newmann Jr JC. Three-dimensional finite-element analysis of finite-thickness fracture specimens. Technical Report NASA TND-8414, NASA Langley Research Center, Hampton, VA 23665, 1977.

34. Green AE, Sneddon IN. The distribution of stress in the neighborhood of a flat elliptical crack in an elastic solid. Proceedings of the Cambridge Philosophical Society 1950; 47:159-164.

35. Irwin GR. The crack extension force for a part-through crack in a plate. ASME, Journal of Applied Mechanics 1962; 29:651-654.

36. Byrd RF, Friedman MD. Handbook of Elliptic Integrals for Engineers \& Scientists. Springer: Berlin; 1971.

37. Li S, Mear ME, Xiao L. Symmetric weak-form integral equation method for three-dimensional fracture analysis. Computer Methods in Applied Mechanics and Engineering 1998; 151:435-459.

38. Benthem JP. The state of stress at the vertex of a quarter-infinite crack in a half space. International Journal of Solids and Structures 1977; 13:479-492.

39. Bazant ZP, Estenssoro, L. Surface singularity and crack propagation. International Journal of Solids and Structures 1979; 15:405-426.

40. Tan CL, Fenner RT. Elastic fracture mechanics analysis by the boundary integral equation method. Proceedings of the Royal Society of London 1979; (369);A:243-260. 